Article

\title{
Starch Components, Starch Properties and Appearance Quality of Opaque Kernels from Rice Mutants
}

\author{
Shun Zhang ${ }^{1,2}$, Zheng $\mathrm{Li}^{1,2}$, Lingshang Lin ${ }^{1,2}$, Long Zhang ${ }^{1,2} \mathbb{1}$ and Cunxu Wei ${ }^{1,2, *(\mathbb{C})}$ \\ 1 Key Laboratory of Crop Genetics and Physiology of Jiangsu Province/Jiangsu Key Laboratory of Crop \\ Genomics and Molecular Breeding, Yangzhou University, Yangzhou 225009, China; \\ zhangshun0918@163.om (S.Z.); yzulizheng@163.com (Z.L.); 18252713442@163.com (L.L.); \\ zhanglong@yzu.edu.cn (L.Z.) \\ 2 Co-Innovation Center for Modern Production Technology of Grain Crops of Jiangsu Province/Joint \\ International Research Laboratory of Agriculture \& Agri-Product Safety of the Ministry of Education, \\ Yangzhou University, Yangzhou 225009, China \\ * Correspondence: cxwei@yzu.edu.cn; Tel.: +86-514-8799-7217
}

Academic Editors: Adam Figiel and Anna Michalska

Received: 13 November 2019; Accepted: 11 December 2019; Published: 13 December 2019

\begin{abstract}
Rice mutants with altered starch components and properties are important genetic resources in rice breeding programmes. In this study, 44 mutants with altered starch components were screened from 135 rice mutants with opaque kernels using a starch-iodine absorption spectrum method, and nine mutants from them were further selected for investigating their starch properties and kernel appearance quality. The results showed that the iodine absorption spectrum parameters, OD620, OD620/550, and $\lambda \max$, could reflect the changes of starch components in rice mutants, and had significantly positive relationships with amylose content and negative relationships with the proportion of short branch-chains of amylopectin. The endosperm starches from nine mutants all showed A-type crystalline structure and similar short-range ordered structure, but had different relative crystallinities. The changes of starch components in mutants not only resulted in the different gelatinization properties of starch but also changed the appearance quality of brown rice kernels. This study provided abundant genetic plants for studying the molecular mechanism of starch synthesis and the quality regulation of rice kernels.
\end{abstract}

Keywords: rice mutant; starch component; physicochemical properties; appearance quality

\section{Introduction}

Rice is one of the most important crops, and provides the staple food for over half the world's population. Starch is the main component of rice endosperm, and its properties greatly influence the eating and cooking qualities of rice and its processing method in industrial use [1-3]. Therefore, the cultivation of rice varieties with different starch properties is one of the important goals in rice breeding. Starch mainly consists of amylose and amylopectin, and the starch components including amylose content (AC) and amylopectin structure determine starch properties [4,5]. According to the AC, rice varieties are usually classified into waxy $(0-2 \%)$, very low (3-9\%), low (10-19\%), intermediate $(20-25 \%)$, and high $(>25 \%)$ amylose classes [6]. The AC plays a crucial role in determining the cooking quality of rice; for example, the cooked rice with low AC exhibits the advantages of smoothness, being refreshing, elasticity, and having a low degree of retrogradation; and cooked rice with high AC is dry and hard [7-9]. In addition, the amylopectin structure also influences the properties of starch. The rice starch with long branch-chains of amylopectin and B-type crystalline contains high resistant starch, 
and has health benefits $[4,5,10]$. Therefore, it is important to cultivate rice varieties with different ACs and amylopectin structures.

In recent years, some novel rice materials with different qualities have been cultivated through traditional cross breeding, chemical or physical mutagenesis breeding, and advanced biotechnological approaches, including transfer DNA or transposon insertion and RNA interference [10-18]. These mutants have significantly different starch properties and expand the uses of rice kernels not only as special food for human health but also as important materials for industry product. Most importantly, these mutants are valuable genetic materials for studying the molecular mechanism of starch synthesis and quality regulation of rice kernels. The mechanism's understanding could accelerate the cultivation of novel rice varieties with improved rice quality [11]. In various kinds of seed mutants, an important phenotype character is that mature kernels are usually opaque and have waxy or floury endosperm. For example, the mutation of genes responsible for protein synthesis, storage, and transport result in floury endosperm $[12,13]$. A mutant with increased aleurone thickness has floury endosperm [14]. Mutating starch synthesis related genes produces floury kernels [15-17]. In addition, the mutation of transcript factor regulating starch and storage protein synthesis genes also results in opaque kernels [18]. The opaque phenotype of kernels is easily detected from rice mutants; however, not all opaque kernels have the changes in starch components. Therefore, it is very necessary, and in theory, possible to rapidly screen mutants with altered starch components and properties from rice mutants with opaque kernels.

In this study, we screened mutants with altered starch components from rice mutants with opaque kernels. Their starch properties and kernel appearance qualities were investigated. This study should provide valuable genetic materials to study the molecular mechanism of starch synthesis, and provide important information for rice quality breeding.

\section{Results and Discussion}

\subsection{Screening Mutants with Altered Starch Components}

Starch contains two main components: amylose and amylopectin. Their contents can be simply detected through measuring their iodine absorption spectra [19]. The iodine absorption spectra of starches from some mutants are shown in Figure 1. The iodine absorption spectrum parameters of starches from 135 rice mutants which are opaque are summarized in Table S1. The $\lambda$ max is the maximum absorption wavelength and is related to the polymerization degree and average chain length of amylose and amylopectin. The OD620 is usually used to assess the AC of starch. The OD620/OD550 can reflect the relative content of longer chain segments in starch [19]. Therefore, the changes of starch components can be detected through analysis of OD620, OD620/OD550, and $\lambda$ max.

Except the starch-iodine colorimetry, the methods for measuring starch components are concanavalin A precipitation, gel permeation chromatograph (GPC), size-exclusion chromatography, near-infrared analysis, and differential scanning calorimetry (DSC) analysis $[4,19,20]$. These methods contain labour-intensive and time-consuming processes and are not suitable for screening mutants with altered starch components from a large number of germplasm samples. In order to rapidly screen mutants with altered starch components from a great quantity of mutants, Agasimani et al. [20] established a simple and rapid single kernel screening method. The method involves transversely cutting the rice transparent kernels in the middle region and dipping the cut end in an optimized iodine solution to estimate the AC in a rice kernel. The time taken for deep blue colouration by the cut end of the kernel after dipping in iodine solution is positively correlated to the AC. However, it is very difficult to judge the colouration of deep blue. In addition, the method is not suitable for kernels with slightly altered AC or with floury endosperm. However, the rice mutants with altered starch components usually have floury endosperm; there is no way to screen the floury mutants with altered starch components using the method of Agasimani et al. [20]. In the present study, the starch components were seen as changes when the differences of OD620, OD620/OD550, and $\lambda$ max between 
mutants and wild type (WT) rice were over $0.02,0.04$, and $7 \mathrm{~nm}$, respectively. According to this standard, 44 rice mutants rapidly screened from 135 rice mutants were found to have the changes of starch components (Table S1). According to the OD620, we further selected nine mutants with OD620 ranging from 0.071 to 0.219 from the 44 mutants to represent the different extents of altered starch components. The iodine absorbance spectra and spectrum parameters of the selected nine mutant starches are summarized in Figure 1 and Table 1. The molecular components and properties of the nine mutant starches were further investigated in the present study.

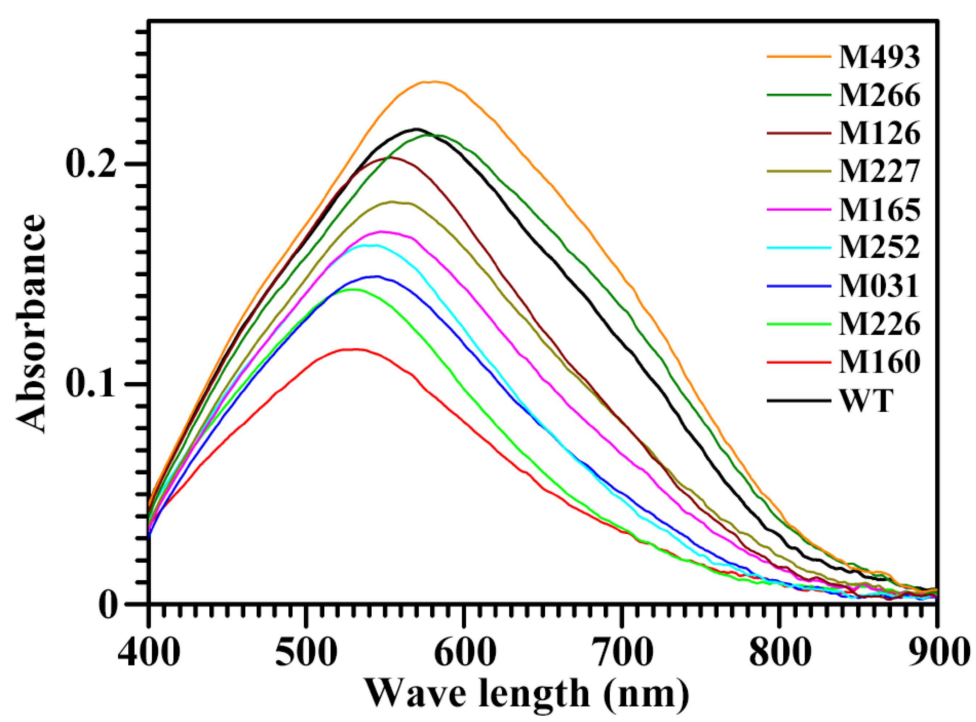

Figure 1. Iodine absorbance spectra of starches from brown rice kernels.

Table 1. Iodine absorption spectrum parameters of starches from brown rice kernels.

\begin{tabular}{ccccc}
\hline Rice Materials & OD620 & OD550 & OD620/OD550 & $\lambda$ max (nm) \\
\hline WT & $0.188 \pm 0.004 \mathrm{e}$ & $0.210 \pm 0.009 \mathrm{f}$ & $0.89 \pm 0.02 \mathrm{~g}$ & $568.2 \pm 1.0 \mathrm{e}$ \\
M160 & $0.071 \pm 0.006 \mathrm{a}$ & $0.112 \pm 0.009 \mathrm{a}$ & $0.63 \pm 0.00 \mathrm{~b}$ & $529.8 \pm 0.7 \mathrm{a}$ \\
M226 & $0.082 \pm 0.009 \mathrm{a}$ & $0.137 \pm 0.015 \mathrm{~b}$ & $0.60 \pm 0.00 \mathrm{a}$ & $529.5 \pm 1.8 \mathrm{a}$ \\
M031 & $0.102 \pm 0.007 \mathrm{~b}$ & $0.148 \pm 0.012 \mathrm{bc}$ & $0.69 \pm 0.01 \mathrm{~d}$ & $542.7 \pm 2.5 \mathrm{~b}$ \\
M252 & $0.107 \pm 0.003 \mathrm{~b}$ & $0.162 \pm 0.006 \mathrm{~cd}$ & $0.66 \pm 0.01 \mathrm{c}$ & $540.4 \pm 0.5 \mathrm{~b}$ \\
M165 & $0.127 \pm 0.005 \mathrm{c}$ & $0.169 \pm 0.007 \mathrm{~cd}$ & $0.75 \pm 0.01 \mathrm{e}$ & $549.9 \pm 0.3 \mathrm{c}$ \\
M227 & $0.145 \pm 0.007 \mathrm{~d}$ & $0.182 \pm 0.010 \mathrm{de}$ & $0.80 \pm 0.01 \mathrm{f}$ & $557.6 \pm 4.0 \mathrm{~d}$ \\
M126 & $0.154 \pm 0.003 \mathrm{~d}$ & $0.202 \pm 0.003 \mathrm{ef}$ & $0.76 \pm 0.00 \mathrm{e}$ & $552.8 \pm 0.6 \mathrm{~cd}$ \\
M266 & $0.195 \pm 0.009 \mathrm{e}$ & $0.202 \pm 0.010 \mathrm{ef}$ & $0.97 \pm 0.01 \mathrm{~h}$ & $579.7 \pm 1.9 \mathrm{f}$ \\
M493 & $0.219 \pm 0.007 \mathrm{f}$ & $0.224 \pm 0.010 \mathrm{f}$ & $0.98 \pm 0.02 \mathrm{~h}$ & $579.5 \pm 3.5 \mathrm{f}$ \\
\hline
\end{tabular}

Data are means \pm SDs, $n=3$. Values in the same column with different letters are significantly different.

\subsection{Starch Components in Brown Kernels of Rice Mutants}

The AC in isolated starch was measured using Megazyme amylose/amylopectin assay kit (Table 2). According to the principle of the kit, the amylose and amylopectin in isolated starch are dissolved completely and quantified. The AC is the percentage of the amylose to both amylose and amylopectin. Therefore, its value is not affected by the starch purity and usually called true AC. The starch from WT rice had $13.3 \% \mathrm{AC}$, and starches from nine selected rice mutants had ACs ranging from $2.2 \%$ to $14.3 \%$. Many opaque mutants have low ACs, especially for the one with the mutated granule-bound starch synthase I gene [21,22].

In order to further confirm the component changes of starch, the isoamylase-debranched starch was analysed using GPC (Figure 2). The three peaks, Peak 1, Peak 2, and Peak 3, of GPC profiles reflect the amylopectin short branch-chains, amylopectin long branch-chains, and amylose molecules, respectively. Their area percentages can assess the contents of starch components, and the area ratio of amylopectin's short to long branch-chains can reflect the branching degree of amylopectin [23]. In the 
present study, starch from WT contained 63.9\% amylopectin short branch-chains, $23.3 \%$ amylopectin long branch-chains, and 12.8\% amylose, and had a 2.75 amylopectin branching degree. However, starches from mutants had amylopectin short branch-chains in proportions from $64.7 \%$ to $75.8 \%$, amylopectin long branch-chains from $20.8 \%$ to $29.1 \%$, amylose from 0 to $13.0 \%$, and amylopectin branching degrees from 2.39 to 3.29 (Table 2). The amylose is synthesized by granule-bound starch synthase I, and the amylopectin is synthesized by the combined actions of soluble starch synthase, starch branching enzyme, and starch debranching enzyme. Mutants with a mutation or expression change of starch-synthesis-related enzyme genes can alter starch components [24,25].

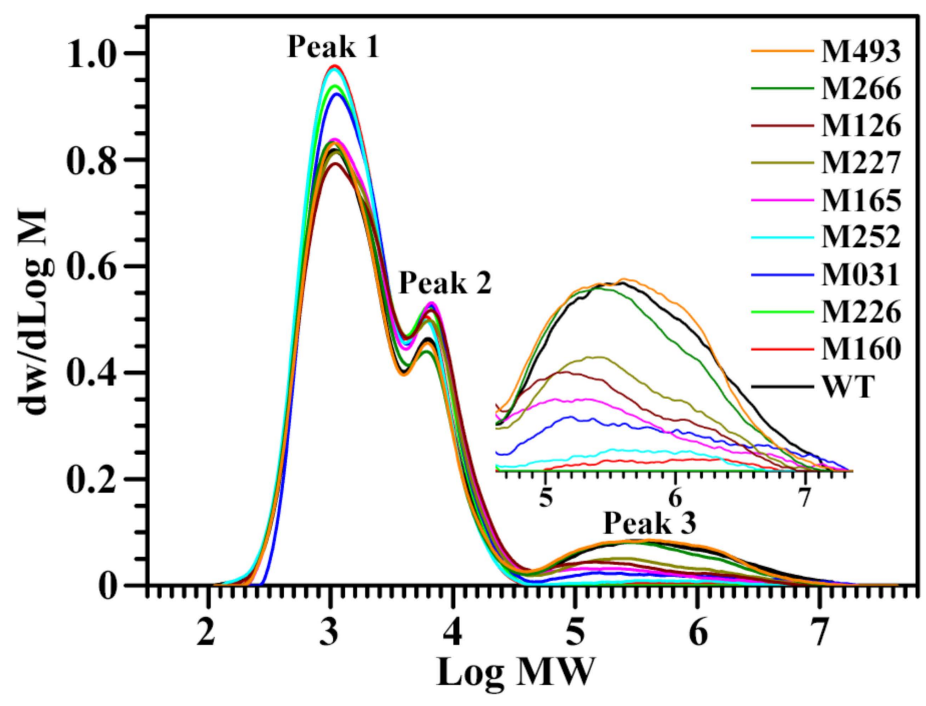

Figure 2. GPC profiles of isoamylase-debranched starches from brown rice kernels.

Table 2. Components of starches from brown rice kernels.

\begin{tabular}{cccccc}
\hline Rice Materials & AC (\%) & AP-S (\%) & AP-L (\%) & AM (\%) & AP-S/L \\
\hline WT & $13.3 \pm 0.2 \mathrm{f}$ & $63.9 \pm 0.5 \mathrm{a}$ & $23.3 \pm 0.5 \mathrm{~b}$ & $12.8 \pm 0.0 \mathrm{e}$ & $2.75 \pm 0.08 \mathrm{~b}$ \\
M160 & $2.2 \pm 0.1 \mathrm{a}$ & $75.8 \pm 0.1 \mathrm{e}$ & $23.1 \pm 0.0 \mathrm{~b}$ & $1.1 \pm 0.1 \mathrm{a}$ & $3.29 \pm 0.01 \mathrm{~d}$ \\
M226 & $3.0 \pm 0.2 \mathrm{ab}$ & $74.6 \pm 0.2 \mathrm{e}$ & $25.4 \pm 0.2 \mathrm{c}$ & $0.0 \pm 0.0 \mathrm{a}$ & $2.94 \pm 0.03 \mathrm{c}$ \\
M031 & $4.1 \pm 0.4 \mathrm{~b}$ & $70.4 \pm 0.3 \mathrm{~d}$ & $25.6 \pm 0.4 \mathrm{c}$ & $4.0 \pm 0.1 \mathrm{~b}$ & $2.74 \pm 0.05 \mathrm{~b}$ \\
M252 & $3.2 \pm 0.1 \mathrm{ab}$ & $69.6 \pm 0.6 \mathrm{~cd}$ & $29.1 \pm 0.0 \mathrm{e}$ & $1.3 \pm 0.6 \mathrm{a}$ & $2.39 \pm 0.02 \mathrm{a}$ \\
M165 & $5.3 \pm 0.4 \mathrm{c}$ & $68.1 \pm 0.1 \mathrm{bc}$ & $27.4 \pm 0.0 \mathrm{~d}$ & $4.5 \pm 0.1 \mathrm{~b}$ & $2.48 \pm 0.00 \mathrm{a}$ \\
M227 & $6.2 \pm 0.6 \mathrm{c}$ & $67.5 \pm 0.7 \mathrm{~b}$ & $24.9 \pm 0.3 \mathrm{c}$ & $7.6 \pm 1.0 \mathrm{~d}$ & $2.71 \pm 0.00 \mathrm{~b}$ \\
M126 & $8.2 \pm 0.3 \mathrm{~d}$ & $66.4 \pm 0.2 \mathrm{~b}$ & $27.7 \pm 0.1 \mathrm{~d}$ & $5.9 \pm 0.0 \mathrm{c}$ & $2.40 \pm 0.02 \mathrm{a}$ \\
M266 & $10.7 \pm 0.5 \mathrm{e}$ & $67.3 \pm 0.9 \mathrm{~b}$ & $20.8 \pm 0.5 \mathrm{a}$ & $11.9 \pm 0.4 \mathrm{e}$ & $3.23 \pm 0.13 \mathrm{~d}$ \\
M493 & $14.3 \pm 0.4 \mathrm{f}$ & $64.7 \pm 0.5 \mathrm{a}$ & $22.4 \pm 0.2 \mathrm{~b}$ & $13.0 \pm 0.3 \mathrm{e}$ & $2.89 \pm 0.05 \mathrm{bc}$ \\
\hline
\end{tabular}

Data are means \pm SDs, $n=3$. Values in the same column with different letters are significantly different. $\mathrm{AC}$, the amylose content in starch measured by amylose/amylopectin assay kit; AP, amylopectin; AP-S, short branch-chains of AP; AP-L, long branch-chains of AP; AP-S/L, ratio of AP-S and AP-L; AM, amylose. The AP-S, $\mathrm{AP}-\mathrm{L}$, and AM are measured through the GPC of isoamylase-debranched starch.

\subsection{Relationships between Iodine Absorption Spectrum Parameters and Starch Components}

In order to reveal whether the changes of iodine absorption spectrum parameters can reflect the changes of starch components, their relationships were analysed (Table 3). The OD620, OD550, OD620/OD550, and $\lambda$ max were significantly positively correlated to AC and negatively correlated to the content of amylopectin short branch-chains $(p<0.01)$. Similar results have been reported in the previous paper [19]. The present study indicated that the mutants with changed starch components could be rapidly detected. Though the Lugol-based coloration assay is fast and reliable in detecting the waxy mutants, it is difficult to detect the non-waxy mutants with changed AC and amylopectin structures using a Lugol-based coloration assay. Therefore, the iodine absorption spectrum method had special potential and usefulness in rapidly screening the non-waxy mutants with changed starch components. 
Table 3. Pearson correlation coefficients between iodine absorption parameters and starch components.

\begin{tabular}{ccccccccc}
\hline & OD620 & OD550 & OD620/550 & $\lambda \max$ & AC & AP-S & AP-L & AM \\
\hline OD550 & $0.964^{* *}$ & & & & & & \\
OD620/550 & $0.975^{* *}$ & $0.890^{* *}$ & & & & & \\
$\lambda$ max & $0.981^{* *}$ & $0.913^{* *}$ & $0.995^{* *}$ & & & & \\
AC & $0.967^{* *}$ & $0.919^{* *}$ & $0.938^{* *}$ & $0.927^{* *}$ & & & \\
AP-S & $-0.899^{* *}$ & $-0.955^{* *}$ & $-0.831^{* *}$ & $-0.858^{* *}$ & $-0.854^{* *}$ & & \\
AP-L & -0.474 & -0.246 & -0.606 & -0.549 & -0.539 & 0.113 & & \\
AM & $0.961^{* *}$ & $0.885^{* *}$ & $0.978^{* *}$ & $0.969^{* *}$ & $0.961^{* *}$ & $-0.851^{* *}$ & -0.618 & \\
AP-S/L & -0.003 & -0.244 & 0.156 & 0.092 & 0.067 & 0.395 & $-0.864^{* *}$ & 0.145 \\
\hline \multicolumn{7}{c}{ The abbreviations of AC, AP-S, AP-L, and AM are explained in Table $2 .{ }^{* *} p<0.01}$. \\
\end{tabular}

Principal component analysis (PCA) is a widely used method to determine the relationships of variables that contribute to differences between physicochemical properties or samples. The iodine absorption spectrum parameters and starch components of ten rice materials were subjected to PCA, and the results are present in Figure 3. The PC1 and PC2 explained $75.7 \%$ and $22.3 \%$ of the overall variation, respectively. For the loading plot of PCA, the properties with curves close to each other on the plot are positively correlated while those with curves in opposite directions are significantly negatively correlated. The loading plot of iodine absorption spectrum parameters and starch components showed that OD550, OD620, AC, $\lambda \max , \mathrm{AM}$, and OD620/550 were highly positive correlated, and they were negatively correlated to the amylopectin short branch-chains (Figure 3A). The score plot of PCA provides an overview of the similarities and differences between the starches from different rice materials. The distance between the locations of any two starches can reflect the degree of the difference and similarity between them. The score plot of PCA showed that starches from different rice mutants had some differences in their starch components (Figure 3B).
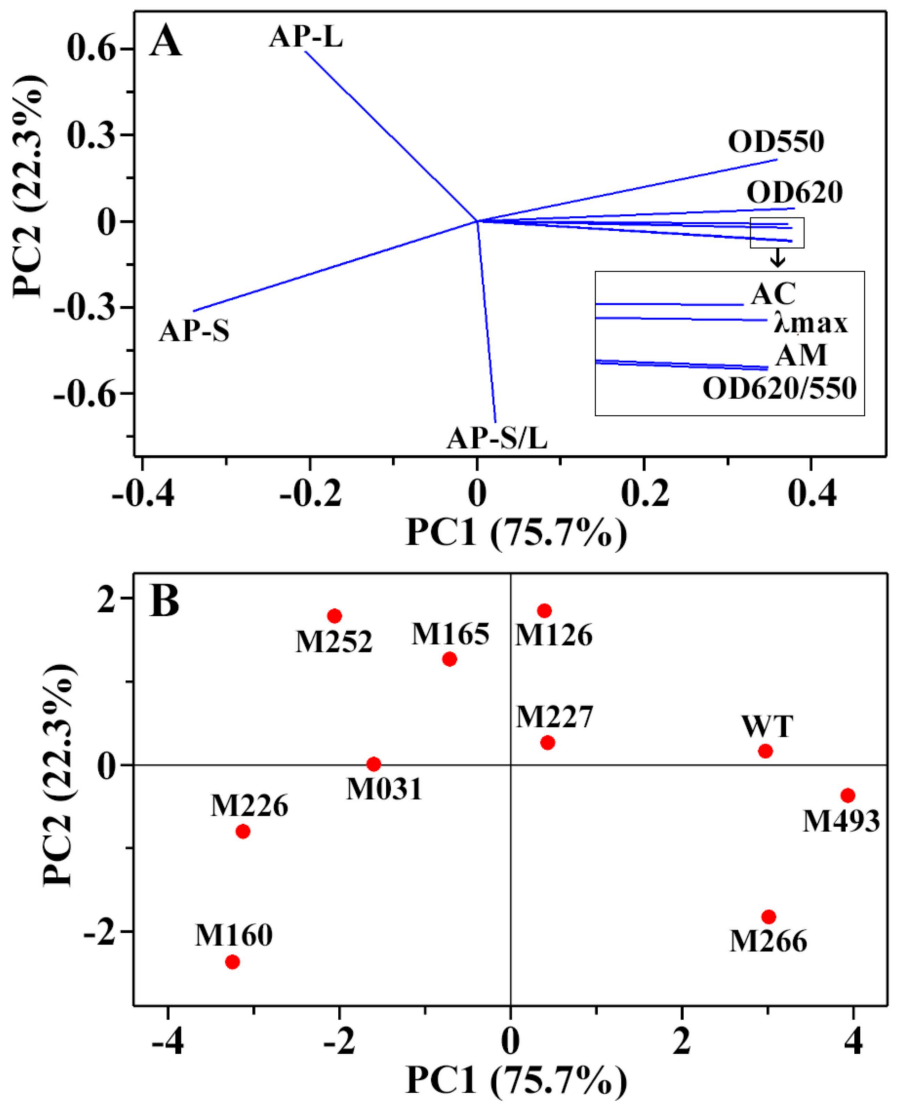

Figure 3. Loading (A) and score plots (B) of principal component analysis based on iodine absorption spectrum parameters and starch components. 


\subsection{Crystalline Structure of Starches from Rice Mutants}

The X-ray diffraction (XRD) patterns of starches are shown in Figure 4. Native starches from different plant sources can be classified into A-, B- or C-type according to their XRD patterns. The starches from normal cereal endosperm have A-type crystallinity; tuber starches show B-type crystallinity; and rhizome and legume starches have C-type crystallinity [26,27]. In the present study, starches from WT rice and its derived nine mutants all had typical A-type XRD patterns with strong diffraction peaks at $15^{\circ}$ and $23^{\circ} 2 \theta$, and an unresolved doublet at $17^{\circ}$ and $18^{\circ} 2 \theta$ (Figure 4 ). The peak at $20^{\circ} 2 \theta$ is the structure of V-type crystallinity and is usually related with amylose-lipid complex [28]. The intensity of peak at $20^{\circ} 2 \theta$ had no significant difference between WT rice and mutant lines except that the M493 line had a slightly high peak intensity (Figure 4). This result might indicate that lipid content in starch was similar between mutant lines and WT rice. Usually, the mutation of starch branching enzyme gene can change the amylopectin structure and increase AC, leading to the change of crystalline structure from A-type to B-type or C-type. However, mutating granule-bound starch synthase I or soluble starch synthase does not change to the crystalline structure of starch, though starch components change significantly [29]. The relative crystallinity ranged from $23.3 \%$ to $31.9 \%$ among WT rice and nine mutants (Figure 4). Usually, the relative crystallinity is significantly negatively correlated with AC and positively correlated with amylopectin short branch-chains [26,27,30]. However, in the present study, Pearson correlation coefficients (data not shown) showed that there was no significant relationship between relative crystallinity and starch components, indicating that some non-starch components in starch granules might influence the relative crystallinity.

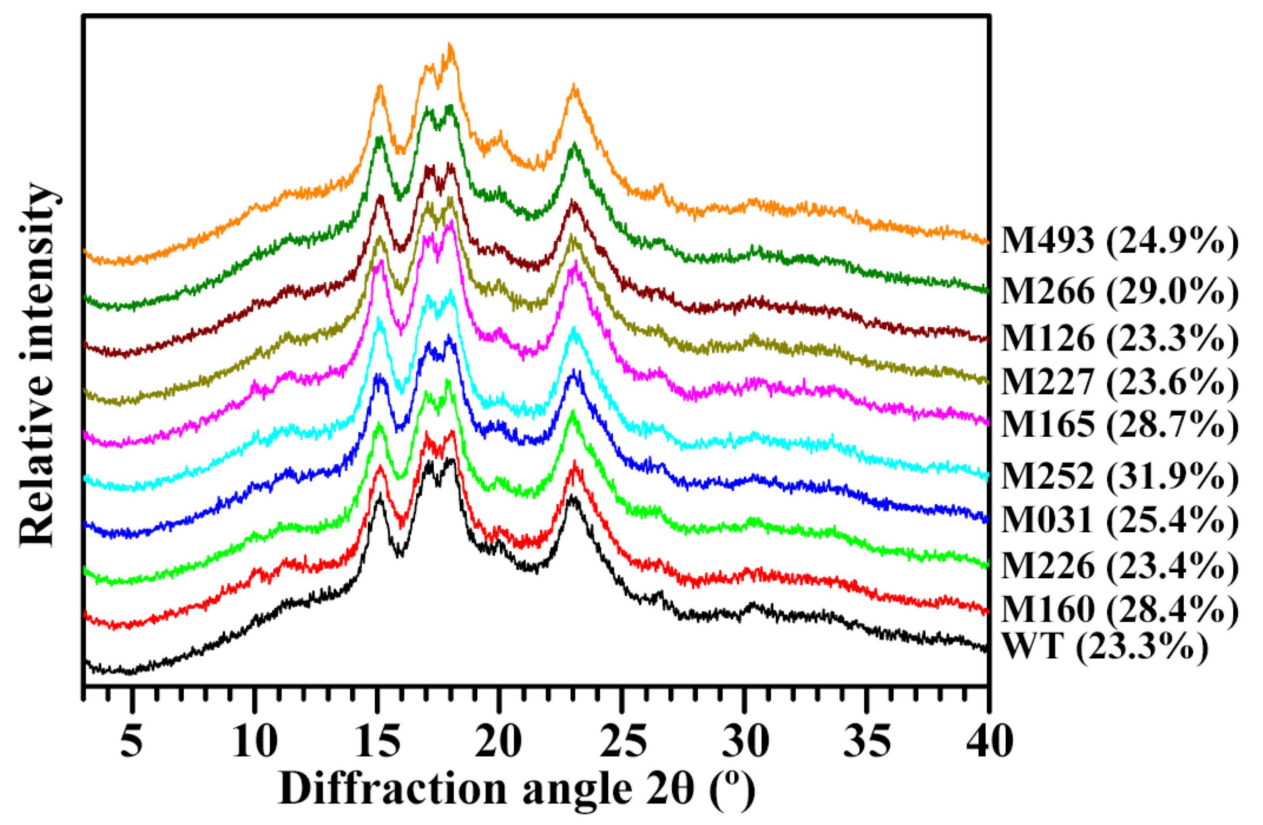

Figure 4. XRD patterns of starches from brown rice kernels. The relative crystallinity of starch is given in the parentheses after the sample name.

\subsection{Short-Range Ordered Structure of Starches from Rice Mutants}

The branch-chains of amylopectin form two types of helices in starch granules. The helices that are packed in short-range order are defined as the double helical order, and the helices that are packed in long-range order are related to the packing of double helices forming crystallinity [31]. The short-range ordered structure of amylopectin can be detected by infrared. The attenuated total reflectance-Fourier transform infrared (ATR-FTIR) is usually used to detect the short-range ordered structure of amylopectin in the external region of starch granule. The ATR-FTIR spectra of starches are shown in Figure 5. For FTIR spectra of starches, the bands at 1045 and $1022 \mathrm{~cm}^{-1}$ have been linked 
with order/crystallinity and amorphous regions in starch, respectively. The ratio of absorbance 1045 to $1022 \mathrm{~cm}^{-1}\left(1045 / 1022 \mathrm{~cm}^{-1}\right)$ is usually used to assess the ordered degree of starch [32]. The FTIR spectra of starches did not have significant differences among WT rice and nine mutants, indicating a similar short-range order structure of amylopectin in the external region of starch granules (Figure 5). The present results agreed with the previous report that the normal maize starch and waxy maize starch have similar ordered degree [32].

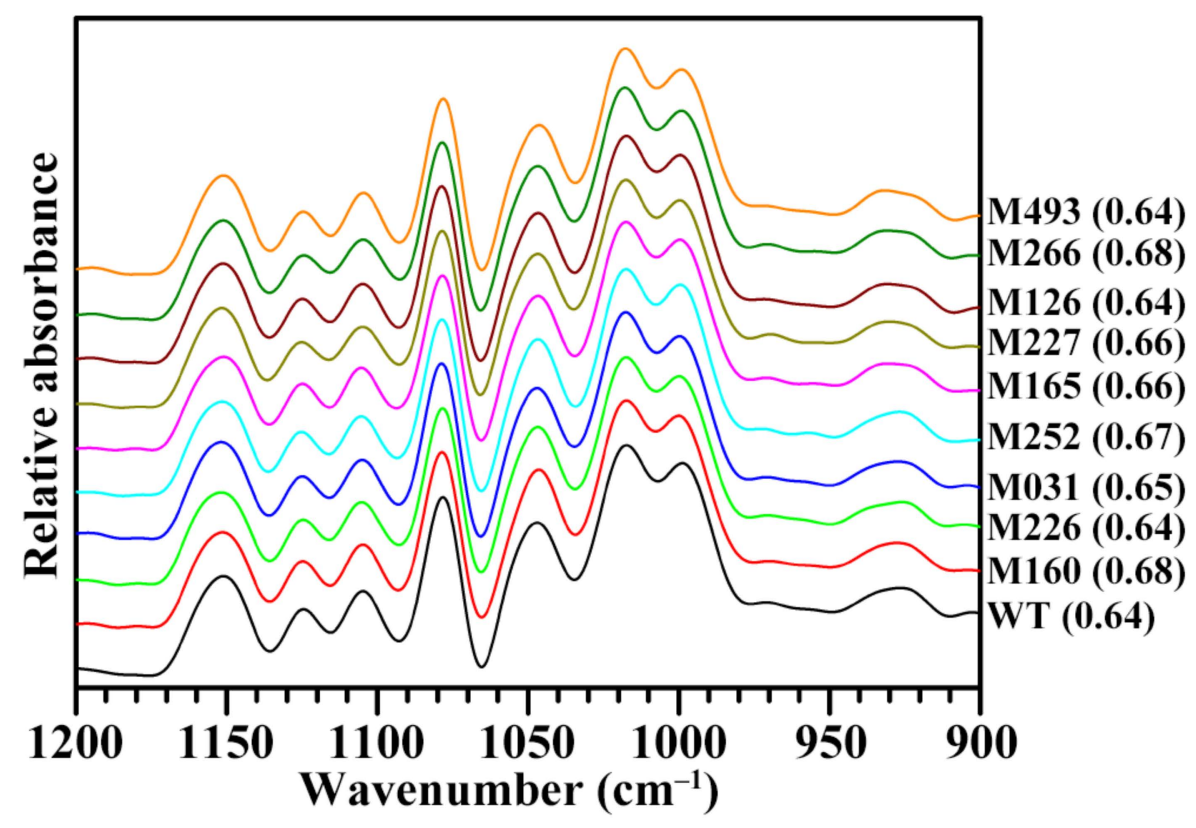

Figure 5. FTIR spectra of starches from brown rice kernels. The short-range ordered degree of starch $\left(1045 / 1022 \mathrm{~cm}^{-1}\right)$ is given in the parentheses after sample name.

\subsection{Thermal Properties of Starches from Rice Mutants}

The thermal properties of starch are important functional properties and determine the applications of starch. The thermal properties of starches are usually determined by DSC, which measures the change of heat involved in starch gelatinization. The gelatinization results in the disruption of double helical and crystalline structure of amylopectin in starch granules. The DSC thermal curves of starches from rice mutants are shown in Figure 6, and their thermal parameters are summarized in Table 4. The starches from different mutants had significantly different gelatinization temperatures and enthalpies. Though the M252 starch had a low AC (1.3\%), it exhibited the highest gelatinization temperature and the lowest gelatinization temperature range and enthalpy. The M266 starch contained the medium AC (11.9\%) but had the lowest gelatinization temperature (Tables 2 and 4 ). From the point of the effect of AC on thermal properties, contradictory results have been reported [30,33-37]. For instance, some papers report that AC is positively correlated with gelatinization temperature $[30,33,34]$, while other references show that waxy starch has higher gelatinization temperature than normal starch [35-37]. The conflicting conclusion is ascribed to the different genetic backgrounds of the rice materials tested [37]. Even in a common rice genetic background with different $W x$ alleles and ACs, the starch with lower AC has a higher gelatinization temperature. This is due to the fact that the lower amylose contributes to the higher relative crystallinity of starch, needing a higher temperature to disrupt it [37]. In fact, the thermal properties of starch are influenced by many factors, such as granule size, AC, amylopectin structure, crystalline structure, and some non-starch components [38]. The change of amylopectin structure affects the thermal properties more than the AC change [35,37]. Among the ten starches, the M252 and M266 starches had the highest (29.1\%) and the lowest (20.8\%) amylopectin long branch-chain contents, respectively (Table 2), and might accordingly need the highest and the lowest temperatures to dissociate them completely (Table 4). The results were in agreement with the previous report [39]. 
In the present study, the different thermal properties of starches from different mutants might be due to amylopectin's molecular structure and AC jointly playing important roles in determining starch gelatinization. In a future work, which gene mutation influenced the starch components and their relations to thermal properties need to be fully clarified.

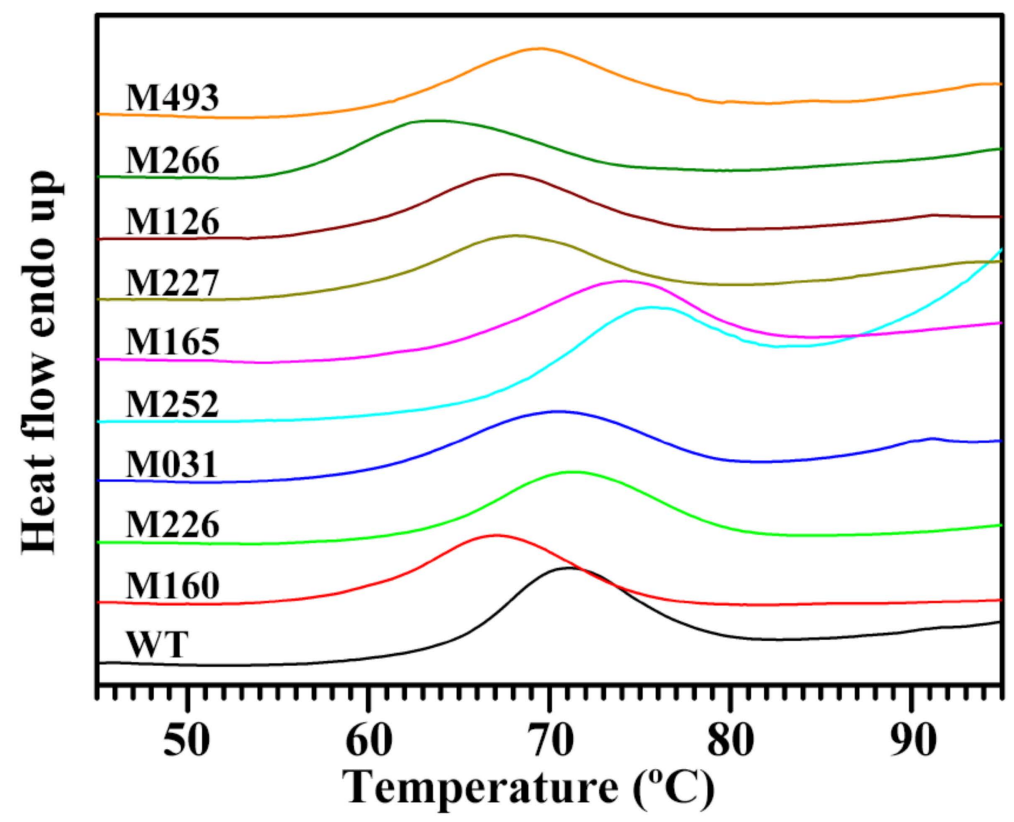

Figure 6. DSC thermal curves of starches from brown rice kernels.

Table 4. Thermal parameters of starches from brown rice kernels.

\begin{tabular}{cccccc}
\hline Rice Materials & To $\left({ }^{\circ} \mathbf{C}\right)$ & $\mathbf{T p}\left({ }^{\circ} \mathrm{C}\right)$ & Tc $\left({ }^{\circ} \mathbf{C}\right)$ & $\Delta \mathbf{T}\left({ }^{\circ} \mathbf{C}\right)$ & $\Delta \boldsymbol{H}(\mathbf{J} / \mathbf{g})$ \\
\hline WT & $64.4 \pm 0.1 \mathrm{e}$ & $71.0 \pm 0.2 \mathrm{e}$ & $78.1 \pm 0.8 \mathrm{c}$ & $13.8 \pm 0.8 \mathrm{a}$ & $10.9 \pm 0.1 \mathrm{~d}$ \\
M160 & $59.1 \pm 0.1 \mathrm{~b}$ & $67.1 \pm 0.1 \mathrm{~b}$ & $75.1 \pm 0.5 \mathrm{ab}$ & $16.0 \pm 0.6 \mathrm{~b}$ & $9.7 \pm 0.6 \mathrm{bcd}$ \\
M226 & $62.9 \pm 0.1 \mathrm{~d}$ & $71.3 \pm 0.1 \mathrm{e}$ & $79.9 \pm 0.3 \mathrm{~d}$ & $17.1 \pm 0.4 \mathrm{bc}$ & $10.4 \pm 0.0 \mathrm{~cd}$ \\
M031 & $60.2 \pm 0.1 \mathrm{c}$ & $70.7 \pm 0.3 \mathrm{e}$ & $79.7 \pm 0.7 \mathrm{~d}$ & $19.5 \pm 0.8 \mathrm{~d}$ & $9.3 \pm 0.5 \mathrm{abcd}$ \\
M252 & $67.9 \pm 0.2 \mathrm{f}$ & $75.7 \pm 0.2 \mathrm{~g}$ & $81.6 \pm 0.6 \mathrm{e}$ & $13.8 \pm 0.8 \mathrm{a}$ & $7.8 \pm 0.3 \mathrm{a}$ \\
M165 & $63.6 \pm 0.2 \mathrm{~d}$ & $74.2 \pm 0.0 \mathrm{f}$ & $81.8 \pm 0.6 \mathrm{e}$ & $18.3 \pm 0.8 \mathrm{~cd}$ & $10.8 \pm 1.0 \mathrm{~d}$ \\
M227 & $59.2 \pm 0.1 \mathrm{~b}$ & $68.3 \pm 0.1 \mathrm{c}$ & $77.1 \pm 0.6 \mathrm{c}$ & $17.9 \pm 0.4 \mathrm{bcd}$ & $8.8 \pm 0.3 \mathrm{abc}$ \\
M126 & $59.5 \pm 0.6 \mathrm{bc}$ & $67.9 \pm 0.4 \mathrm{c}$ & $76.4 \pm 0.1 \mathrm{bc}$ & $16.9 \pm 0.6 \mathrm{bc}$ & $8.5 \pm 0.8 \mathrm{ab}$ \\
M266 & $55.7 \pm 0.1 \mathrm{a}$ & $63.9 \pm 0.3 \mathrm{a}$ & $74.2 \pm 0.1 \mathrm{a}$ & $18.5 \pm 0.1 \mathrm{~cd}$ & $8.6 \pm 0.2 \mathrm{abc}$ \\
M493 & $60.2 \pm 0.1 \mathrm{c}$ & $69.5 \pm 0.1 \mathrm{~d}$ & $78.0 \pm 0.0 \mathrm{c}$ & $17.8 \pm 0.1 \mathrm{bcd}$ & $8.6 \pm 0.3 \mathrm{abc}$ \\
\hline
\end{tabular}

Data are means \pm SDs, $n=3$. Values in the same column with different letters are significantly different. $\mathrm{To}$, gelatinization onset temperature; $\mathrm{Tp}$, gelatinization peak temperature; $\mathrm{Tc}$, gelatinization conclusion temperature; $\Delta \mathrm{T}$, gelatinization temperature range; $\Delta \mathrm{H}$, gelatinization enthalpy.

\subsection{Appearance Quality of Brown Rice Kernels}

The unhulled mature grains are shown in Figure 7A. No significant differences were observed except for the slight change of grain size. The brown rice kernels were observed under transmitted light. The WT kernels were transparent, but the mutant kernels showed an opaque phenotype (Figure 7B). In order to reveal the reasons for poor transparency of kernels from mutants, the cross fractured planes of brown rice kernels were observed under stereoscopic microscope (Figure 7C). The M160, M226, and M252 kernels were waxy and opaque, the other mutants had floury endosperms. It has been widely reported that the waxy rice kernels with ACs below $2 \%$ result from a deficiency of granule-bound starch synthase I and exhibit a typically waxy phenotype [40]. Recently, Zhang et al. [41] reported that the waxy phenotype of a dry kernel is due to the cavities in the centre of starch granules. The kernels with altered starch components in non-waxy rice mutants usually exhibit floury phenotypes, due to 
the fact that the synthesis of starch is influenced and starch granules are loosely packed in endosperm cells [42]. In the present study, the morphology observations of brown kernels of rice mutants were in agreement with the results of starch component (Table 2). The M160, M226, and M252 lines had ACs below 2.0\%, and exhibited waxy kernels. The M031, M165, M227, M126, M266, and M493 lines had AC changes from $4.0 \%$ to $13.0 \%$ and exhibited floury kernels. The dehulled brown rice kernels were measured for their length, width, thickness, and weight. Some mutant kernels had significantly lower kernel length, width, thickness, and weight values than WT kernels, but some mutant kernels had similar kernel shapes and weights to WT kernels (Table 5).

Compared with floury endosperm mutants with slightly changed starch components due to the mutation of storage protein synthesis or aleurone cell development [12-14], the newly screened non-waxy floury endosperm M031, M165, M227, M126, M266, and M493 lines had significantly changed starch components, starch properties, and appearance quality of kernel. Some rice floury endosperm materials resulting from the mutation or regulating expression of starch synthesis related genes have been reported; their starch components, starch properties and kernel quality significantly change [10,11,15-18]. However, it is very complicated to understand and regulate the starch synthesis and kernel quality, and more mutants are needed to reveal the regulation mechanism. Therefore, the newly selected non-waxy mutants with significantly changed starch components were valuable genetic materials for further investing the molecular mechanism of starch synthesis and the quality regulation of rice kernels.
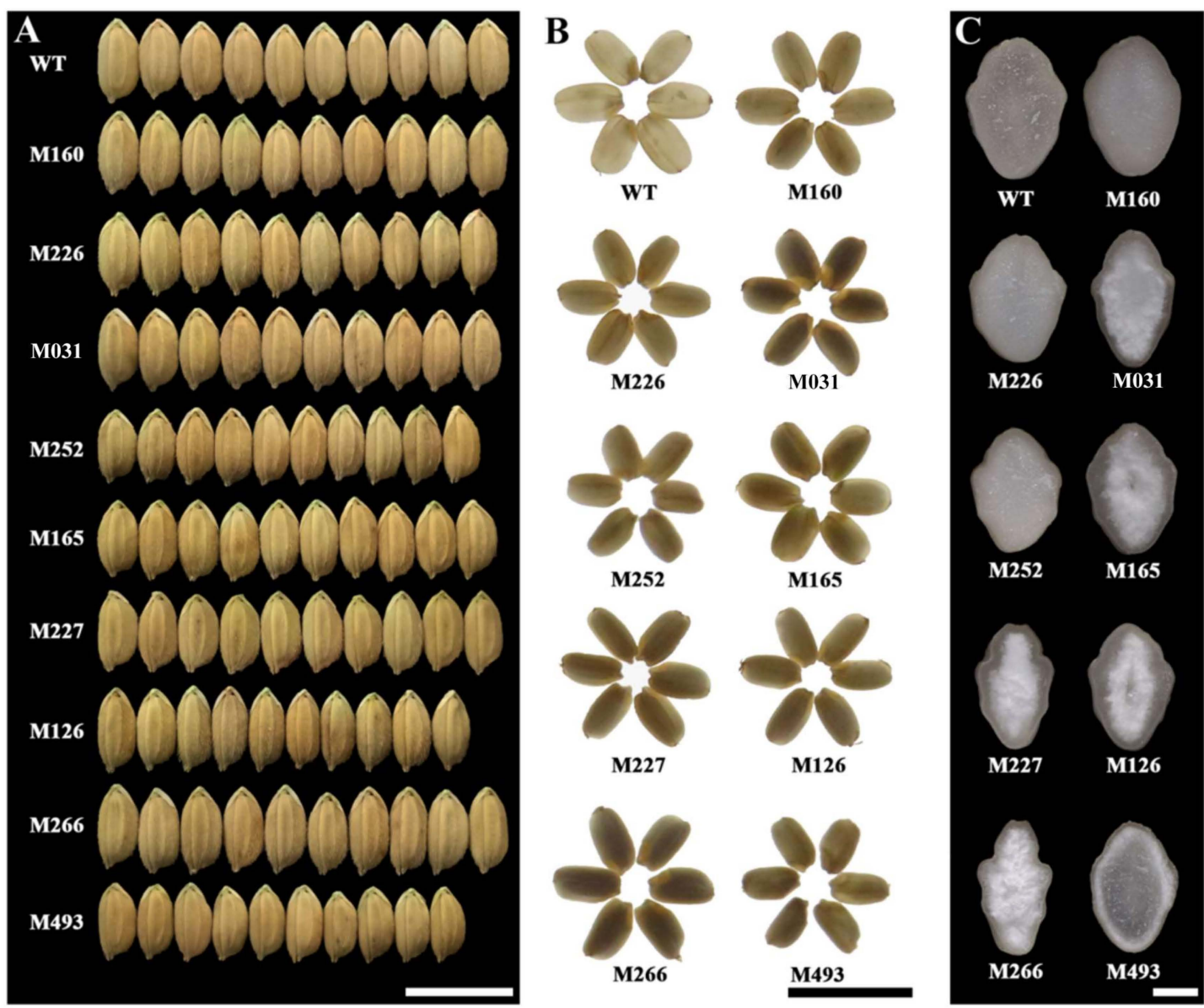

Figure 7. Morphologies of grains (A), brown rice kernels (B), and cross sections from the mid-regions of brown rice kernels. Scale bar $=1 \mathrm{~cm}$ for $(\mathbf{A}, \mathbf{B})$ and $1 \mathrm{~mm}$ for $(\mathbf{C})$. 
Table 5. Length, width, thickness, and weight values of brown rice kernels.

\begin{tabular}{ccccc}
\hline Rice Materials & Length $(\mathbf{m m})$ & Width $\mathbf{( m m )}$ & Thickness $(\mathbf{m m})$ & Weight $(\mathbf{g} / \mathbf{1 0 0}$ Kernels) \\
\hline WT & $5.11 \pm 0.12 \mathrm{~d}$ & $3.02 \pm 0.07 \mathrm{~d}$ & $2.10 \pm 0.07 \mathrm{ef}$ & $2.23 \pm 0.01 \mathrm{~h}$ \\
M160 & $4.77 \pm 0.13 \mathrm{~b}$ & $2.92 \pm 0.09 \mathrm{bc}$ & $2.08 \pm 0.04 \mathrm{ef}$ & $2.01 \pm 0.02 \mathrm{f}$ \\
M226 & $5.14 \pm 0.16 \mathrm{~d}$ & $2.88 \pm 0.11 \mathrm{~b}$ & $2.09 \pm 0.06 \mathrm{ef}$ & $2.22 \pm 0.03 \mathrm{~h}$ \\
M031 & $4.80 \pm 0.11 \mathrm{~b}$ & $2.99 \pm 0.09 \mathrm{~cd}$ & $2.11 \pm 0.06 \mathrm{f}$ & $2.11 \pm 0.00 \mathrm{~g}$ \\
M252 & $4.71 \pm 0.06 \mathrm{~b}$ & $2.89 \pm 0.08 \mathrm{~b}$ & $2.04 \pm 0.05 \mathrm{de}$ & $1.95 \pm 0.01 \mathrm{e}$ \\
M165 & $4.72 \pm 0.17 \mathrm{~b}$ & $2.88 \pm 0.09 \mathrm{~b}$ & $1.94 \pm 0.19 \mathrm{bc}$ & $1.65 \pm 0.02 \mathrm{~b}$ \\
M227 & $4.95 \pm 0.08 \mathrm{c}$ & $2.96 \pm 0.07 \mathrm{bcd}$ & $1.98 \pm 0.07 \mathrm{~cd}$ & $1.81 \pm 0.01 \mathrm{~d}$ \\
M126 & $4.94 \pm 0.13 \mathrm{c}$ & $2.77 \pm 0.16 \mathrm{a}$ & $1.93 \pm 0.11 \mathrm{bc}$ & $1.81 \pm 0.01 \mathrm{~d}$ \\
M266 & $5.28 \pm 0.18 \mathrm{e}$ & $2.95 \pm 0.25 \mathrm{~cd}$ & $1.64 \pm 0.10 \mathrm{a}$ & $1.59 \pm 0.00 \mathrm{a}$ \\
M493 & $4.59 \pm 0.12 \mathrm{a}$ & $2.75 \pm 0.10 \mathrm{a}$ & $1.89 \pm 0.09 \mathrm{~b}$ & $1.69 \pm 0.02 \mathrm{c}$ \\
\hline
\end{tabular}

Data are means \pm SDs, $n=3$. Values in the same column with different letters are significantly different.

\section{Materials and Methods}

\subsection{Plant Materials}

The rice mutants with opaque kernels were derived from a japonica rice (Oryza sativa L.) variety (WT), Kitaake, through ${ }^{60} \mathrm{Co}$ gamma irradiation [43]. These mutants were homozygotes and self-pollinated for three generations. The WT rice and mutants were planted simultaneously in an experiment field of Yangzhou University, Yangzhou, China. The mature grains were dehulled, and the brown kernels were used as plant materials in this study.

\subsection{Isolation of Starch from Brown Rice Kernels}

Starch was isolated from brown rice kernels following the method of Lin et al. [19] with some modifications. Briefly, mature and dry kernels were extensively ground into powder with a mortar, and then were ground in deionized water. The starch-water slurry was filtered through 150 and $75 \mu \mathrm{m}$ sieve, and centrifuged. The starch precipitation was washed 3 times using deionized water, and the top dirty materials were carefully removed. The starch was dehydrated two times using anhydrous ethanol, dried at $40{ }^{\circ} \mathrm{C}$, and ground through $150 \mu \mathrm{m}$ sieve. The samples were stored at $4{ }^{\circ} \mathrm{C}$.

\subsection{Determination of Starch-Iodine Absorption Spectrum}

The iodine absorption spectrum of starch was measured exactly following the method of Lin et al. [19]. Briefly, starch was dissolved in dimethyl sulfoxide (DMSO) containing 10\% $6 \mathrm{M}$ urea, and coloured with $\mathrm{I}_{2} / \mathrm{KI}$. The sample was scanned from 400 to $900 \mathrm{~nm}$ using a spectrophotometer (Ultrospec 6300 pro, Amersham Biosciences, Cambridge, UK).

\subsection{Determination of AC in Starch}

The AC in isolated starch was measured using an amylose/amylopectin assay kit (Megazyme, Bray, Ireland).

\subsection{Determination of Molecular Weight Distribution of Starch}

The molecular weight distribution of starch was analysed using GPC following the method of Lin et al. [19]. Briefly, starch was deproteinized first with protease and sodium bisulfite, then dissolved in hot water, and finally, debranched finally using isoamylase. The debranched starch was analysed using a GPC system (PL-GPC 220, Agilent Technologies UK Limited, Shropshire, UK) with three columns (PL110-6100, 6300, 6526) and a differential refractive index detector. 


\subsection{Analysis of Crystalline Structure of Starch}

The crystalline structure of starch was measured using X-ray diffractometer (D8, Bruker, Karlsruhe, Germany) following the method of Lin et al. [4]. The test conditions included the voltage of $40 \mathrm{kV}$, electric current of $200 \mathrm{~mA}$, step size of $0.02^{\circ}$, and scanning range from $4^{\circ}$ to $40^{\circ} 2 \theta$.

\subsection{Analysis of Short-Range Ordered Structure of Starch}

The short-range ordered structure of starch was measured using an attenuated total reflectance-Fourier transform infrared (ATR-FTIR) spectrometer (7000, Varian, Santa Clara, CA, USA) following the method of Lin et al. [4]. The test conditions included the scanning range from 400 to $4000 \mathrm{~cm}^{-1}$, resolution of $4 \mathrm{~cm}^{-1}$, and scan times of 64 . The original spectrum was corrected by a baseline from 1200 to $800 \mathrm{~cm}^{-1}$, and deconvoluted using a Resolutions Pro with resolution enhancement factor of 1.9 and peak half-width of $1.9 \mathrm{~cm}^{-1}$.

\subsection{Determination of Thermal Properties of Starch}

Five $\mathrm{mg}$ of starch and $15 \mu \mathrm{L}$ deionized water were mixed and sealed in an aluminium pan. The sample was equilibrated at room temperature for $2 \mathrm{~h}$, and then analysed using a differential scanning calorimeter (200-F3, Netzsch, Selb, Germany). The test conditions included a heating rate of $10^{\circ} \mathrm{C} / \mathrm{min}$ and a temperature range from 20 to $130^{\circ} \mathrm{C}$.

\subsection{Measurement of Appearance Quality of Brown Rice Kernels}

The hulled grains and dehulled brown rice kernels were photographed with a digital camera (IXUS 750, Canon, Tokyo, Japan). The cross section of brown rice kernel at the middle region was photographed using a Stereo Microscope (EZ4W, Leica, Wetzlar, Germany). The lengths, widths, and thicknesses of 30 brown rice kernels were measured using an electronic digital calliper (0-150, Shanghai Shenhan Measuring Tools Co., Shanghai, China). The 100 brown rice kernels were weighed and repeated three times.

\subsection{Statistical Analysis}

The data were analysed using the SPSS 16.0 Statistical Software Program, and the one-way analysis of variance was evaluated using Tukey's test. The principle component analysis was carried out using Minitab Version 16.0 Software (IBM Company, Chicago, IL, USA).

\section{Conclusions}

The starch-iodine absorption spectrum parameters: OD620, OD620/550, and $\lambda$ max, could reflect the component changes of starch. Forty-four rice mutants with ACs from $2.2 \%$ to $14.3 \%$ were rapidly screened from 135 rice mutants with opaque kernels according to the changes of starch-iodine absorption spectrum parameters. The screened mutants had significantly different starch molecular weight distributions but exhibited the same A-type crystallinity and similar short-range ordered structures. The mutants with different starch components had different thermal properties of starch, and exhibited significant differences in length, width, thickness, and weight of their brown rice kernels. The newly selected non-waxy floury mutants are valuable genetic materials for further investing the molecular mechanism of starch synthesis and the quality regulation of rice kernels.

Supplementary Materials: The following are available online. Table S1: Iodine absorbance spectrum parameters of starches from brown rice kernels.

Author Contributions: C.W. conceived the study and designed the experiments. S.Z., Z.L., L.L., and L.Z. performed the experiments. S.Z. and C.W. analysed the data and wrote the manuscript.

Funding: This research was supported by the Talent Project of Yangzhou University and the Priority Academic Program Development of Jiangsu Higher Education Institutions. 
Conflicts of Interest: The authors declare no conflict of interest. The founding sponsors had no role in the design of the study; in the collection, analyses, or interpretation of data; in the writing of the manuscript; or in the decision to publish the results.

\section{References}

1. Tian, Z.; Qian, Q.; Liu, Q.; Yan, M.; Liu, X.; Yan, C.; Liu, G.; Gao, Z.; Tang, S.; Zeng, D.; et al. Allelic Diversities in Rice Starch Biosynthesis Lead to A Diverse Array of Rice Eating and Cooking Qualities. Proc. Natl. Acad. Sci. USA 2009, 22, 21760-21765. [CrossRef] [PubMed]

2. Umemoto, T.; Horibata, T.; Aoki, N.; Hiratsuka, M.; Yano, M.; Inouchi, N. Effects of Variations in Starch Synthase on Starch Properties and Eating Quality of Rice. Plant. Prod. Sci. 2008, 11, 472-480. [CrossRef]

3. Fan, X.Y.; Guo, M.; Li, R.D.; Yang, Y.H.; Liu, M.; Zhu, Q.; Tang, S.Z.; Gu, M.H.; Xu, R.G.; Yan, C.J. Allelic Variations in the Soluble Starch Synthase II Gene Family Result in Changes of Grain Quality and Starch Properties in Rice (Oryza sativa L.). J. Agric. Sci. 2017, 155, 129-140. [CrossRef]

4. Lin, L.; Guo, K.; Zhang, L.; Zhang, C.; Liu, Q.; Wei, C. Effects of Molecular Compositions on Crystalline Structure and Functional Properties of Rice Starches with Different Amylopectin Extra-Long Chains. Food. Hydrocolloid. 2019, 88, 137-145. [CrossRef]

5. Lin, L.; Pan, T.; Liu, Q.; Wei, C. Cooking, Morphological, Mechanical and Digestion Properties of Cooked Rice with Suppression of Starch Branching Enzymes. Int. J. Biol. Macromol. 2019, 137, 187-196. [CrossRef]

6. Kumar, I.; Khush, G.S. Genetic Analysis of Different Amylose Levels in Rice. Crop. Sci. 1987, $27,1167-1172$. [CrossRef]

7. Noda, T.; Nishiba, Y.; Sato, T.; Suda, I. Properties of Starches from Several Low-Amylose Rice Cultivars. Cereal. Chem. 2003, 80, 193-197. [CrossRef]

8. Li, Q.F.; Huang, L.C.; Chu, R.; Li, J.; Jiang, M.Y.; Zhang, C.Q.; Fan, X.L.; Yu, H.X.; Gu, M.H.; Liu, Q.Q. Down-Regulation of SSSII-2 Gene Expression Results in Novel Low-Amylose Rice with Soft, Transparent Grains. J. Agric. Food. Chem. 2018, 66, 9750-9760. [CrossRef]

9. Tao, K.; Yu, W.; Prakash, S.; Gilbert, R.G. High-Amylose Rice: Starch Molecular Structural Features Controlling Cooked Rice Texture and Preference. Carbohyd. Polym. 2019, 219, 251-260. [CrossRef]

10. Zhu, L.; Gu, M.; Meng, X.; Cheung, S.C.K.; Yu, H.; Huang, J.; Sun, Y.; Shi, Y.; Liu, Q. High-Amylose Rice Improves Indices of Animal Health in Normal and Diabetic Rats. Plant. Biotechnol. J. 2012, 10, 353-362. [CrossRef]

11. Satoh, H.S.; Nishi, A.; Fujita, N.; Kubo, A.; Nakamura, Y.; Kawasaki, T.; Okita, T.W. Isolation and Characterization of Starch Mutants in Rice. J. Appl. Glycosci. 2003, 50, 225-230. [CrossRef]

12. Wang, Y.H.; Liu, F.; Ren, Y.L.; Wang, Y.; Liu, X.; Long, W.; Wang, D.; Zhu, J.; Zhu, X.; Jing, R.; et al. GOLGI TRANSPORT 1B Regulates Protein Export from the Endoplasmic Reticulum in Rice Endosperm Cells. Plant. Cell. 2016, 28, 2850-2865. [CrossRef] [PubMed]

13. Ren, Y.L.; Wang, Y.H.; Liu, F.; Zhou, K.; Ding, Y.; Zhou, F.; Wang, Y.; Liu, K.; Gan, L.; Ma, W.; et al. GLUTELIN PRECURSOR ACCUMULATION3 Encodes a Regulator of Post-Golgi Vesicular Traffic Essential for Vacuolar Protein Sorting in Rice Endosperm. Plant. Cell. 2014, 26, 410-425. [CrossRef] [PubMed]

14. Liu, J.; Wu, X.; Yao, X.; Yu, R.; Larkin, P.J.; Liu, C.M. Mutations in the DNA Demethylase OsROS1 Result in a Thickened Aleurone and Improved Nutritional Value in Rice Grains. Proc. Natl. Acad. Sci. USA 2018, 115, 11327-11332. [CrossRef] [PubMed]

15. Nakagami, T.; Yoshihara, H.; Nakamura, T.; Utsumi, Y.; Sawada, T.; Fujita, N.; Satoh, H.; Nakamura, Y. Biochemical Analysis of New-Type Mutants of Japonica Rice that Accumulate Water-Soluble $\alpha$-glucans in the Endosperm but Retain Full Starch Debranching Enzyme Activities. Starch 2017, 69, 1600159. [CrossRef]

16. Asai, H.; Abe, N.; Matsushima, R.; Crofts, N.; Oitome, N.F.; Nakamura, Y.; Fujita, N. Deficiencies in Both Starch Synthase IIIa and Branching Enzyme IIb Lead to a Significant Increase in Amylose in SSIIa-Inactive Japonica Rice Seeds. J. Exp. Bot. 2014, 65, 5497-5507. [CrossRef] [PubMed]

17. Lee, S.K.; Hwang, S.K.; Han, M.; Eom, J.S.; Kang, H.G.; Han, Y.; Choi, S.B.; Cho, M.H.; Bhoo, S.H.; An, G.; et al. Identification of the ADP-glucose Pyrophosphorylase Isoforms Essential for Starch Synthesis in the Leaf and Seed Endosperm of Rice (Oryza sativa L.). Plant. Mol. Biol. 2007, 65, 531-546. [CrossRef]

18. Wang, J.C.; Xu, H.; Zhu, Y.; Liu, Q.Q.; Cai, X.L. OsbZIP58, A Basic Leucine Zipper Transcription Factor, Regulates Starch Biosynthesis in Rice Endosperm. J. Exp. Bot. 2013, 64, 3453-3466. [CrossRef] 
19. Lin, L.; Zhang, Q.; Zhang, L.; Wei, C. Evaluation of the Molecular Structural Parameters of Normal Rice Starch and their Relationships with its Thermal and Digestion Properties. Molecules 2017, 22, 1526. [CrossRef]

20. Agasimani, S.; Selvakumar, G.; Joel, A.J.; Ram, S.G. A Simple and Rapid Single Kernel Screening Method To Estimate Amylose Content in Rice Grains. Phytochem. Anal. 2013, 24, 569-573. [CrossRef]

21. Liu, L.; Ma, X.; Liu, S.; Zhu, C.; Jiang, L.; Wang, Y.; Shen, Y.; Ren, Y.; Dong, H.; Chen, L.; et al. Identification and Characterization of a Novel Waxy Allele from a Yunnan Rice Landrace. Plant. Mol. Biol. 2009, 71, 609-626. [CrossRef] [PubMed]

22. Shahid, S.; Begum, R.; Razzaque, S.; Seraj, Z.I. Variability in Amylose Content of Bangladeshi Rice Cultivars due to Unique SNPs in Waxy Allele. J. Cereal. Sci. 2016, 71, 1-9. [CrossRef]

23. Wang, Y.J.; White, P.; Pollak, L.; Jane, J. Characterization of Starch Structures of 17 Maize Endosperm Mutant Genotypes with Oh43 Inbred Line Background. Cereal. Chem. 1993, 70, 171.

24. Crofts, N.; Abe, N.; Oitome, N.F.; Matsushima, R.; Hayashi, M.; Tetlow, I.J.; Emes, M.J.; Nakamura, Y.; Fujita, N. Amylopectin Biosynthetic Enzymes from Developing Rice Seed Form Enzymatically Active Protein Complexes. J. Exp. Bot. 2015, 66, 4469-4482. [CrossRef] [PubMed]

25. Crofts, N.; Lizuka, Y.; Abe, N.; Miura, S.; Kikuchi, K.; Matsushima, R.; Fujita, N. Rice Mutants Lacking Starch Systhase I or Branching Enzyme Ilb Activity Altered Starch Biosynthetic Protein Complexes. Front. Plant. Sci. 2018, 9, 1817. [CrossRef]

26. Cheetham, N.W.H.; Tao, L. Variation in Crystalline Type with Amylose Content in Maize Starch Granules: An X-ray Powder Diffraction Study. Carbohyd. Polym. 1998, 36, 277-284. [CrossRef]

27. He, W.; Wei, C. Progress in C-type starches from different plant sources. Food. Hydrocolloid. 2017, 73, 162-175. [CrossRef]

28. Dries, D.M.; Gomand, S.V.; Delcour, J.A.; Goderis, B. V-type Crystal Formation in Starch by Aqueous Ethanol Treatment: The effect of amylose degree of polymerization. Food. Hydrocolloid. 2016, 61, 649-661. [CrossRef]

29. Wang, J.; Hu, P.; Chen, Z.; Liu, Q.; Wei, C. Progress in High-Amylose Cereal Crops through Inactivation of Starch Branching Enzymes. Front. Plant. Sci. 2017, 8, 469. [CrossRef]

30. Cai, J.; Man, J.; Huang, J.; Liu, Q.; Wei, X.; Wei, C. Relationship between Structure and Functional Properties of Normal Rice Starches with Different Amylose Contents. Carbohyd. Polym. 2015, 125, 35-44. [CrossRef]

31. Atichokudomchai, N.; Varavinit, S.; Chinachoti, P. A Study of Ordered Structure in Acid-Modified Tapioca Starch by ${ }^{13} \mathrm{C} C \mathrm{CP} / \mathrm{MAS}$ Solid-State NMR. Carbohyd. Polym. 2004, 58, 383-389. [CrossRef]

32. Sevenou, O.; Hill, S.E.; Farhat, I.A.; Mitchell, J.R. Organisation of the External Region of the Starchgranule as Determined by Infrared Spectroscopy. Int. J. Biol. Macromol. 2002, 31, 79-85. [CrossRef]

33. Park, I.M.; Ibáñez, A.M.; Zhang, F.; Shoemaker, C.F. Gelatinization and Pasting Properties of Waxy and Non-Waxy Rice Starches. Starch 2007, 59, 388-396. [CrossRef]

34. Chung, H.J.; Liu, Q.; Lee, L.; Wei, D. Relationship between the Structure, Physicochemical Properties and in vitro Digestibility of Rice Starches with Different Amylose Contents. Food. Hydrocolloid. 2011, 25, 968-975. [CrossRef]

35. Kubo, A.; Akdogan, G.; Nakaya, M.; Shojo, A.; Suzuki, S.; Satoh, H.; Kitamura, S. Structure, Physical, and Digestive Properties of Starch from wx ae Double-Mutant Rice. J. Agric. Food. Chem. 2010, 58, 4463-4469. [CrossRef]

36. Zhu, L.J.; Liu, Q.Q.; Wilson, J.D.; Gu, M.H.; Shi, Y.C. Digestibility and Physicochemical Properties of Rice (Oryza sativa L.) Flours and Starches Differing in Amylose Content. Carbohyd. Polym. 2011, 86, 1751-1759. [CrossRef]

37. Teng, B.; Zhang, Y.; Du, S.; Wu, J.; Li, Z.; Luo, Z.; Yang, J. Crystalline, Thermal and Swelling Properties of Starches from Single-Segment Substitution Lines with Different Wx Alleles in Rice (Oryza sativa L.). J. Sci. Food. Agric. 2017, 97, 108-114. [CrossRef]

38. Cai, J.; Cai, C.; Man, J.; Zhou, W.; Wei, C. Structural and Functional Properties of C-type Starches. Carbohyd. Polym. 2014, 101, 289-300. [CrossRef]

39. Yamin, F.F.; Lee, M.; Pollak, L.M.; White, P.J. Thermal Properties of Starch in Corn Variants Isolated after Chemical Mutagenesis of Inbred Lines B73. Cereal. Chem. 1999, 76, 175-181. [CrossRef]

40. Lin, B.N.; Hsu, Y.C.; Kuo, S.C.; Lin, Y.R.; Wu, Y.P.; Kuo, C.W. Novel Wx Alleles Induced by Chemical Mutagens in Rice, Oryza sativa L. Plant. Breeding. 2017, 136, 206-213. [CrossRef]

41. Zhang, L.; Zhao, L.; Zhang, J.; Cai, X.; Liu, Q.; Wei, C. Relationships between Transparency, Amylose Content, Starch Cavity, and Moisture of Brown Rice Kernels. J. Cereal. Sci. 2019, 90, 102854. [CrossRef] 
42. Wu, Y.; Pu, C.; Lin, H.; Huang, H.; Huang, Y.; Hong, C.; Chang, M.; Lin, Y. Three Novel Alleles of FLOURY ENDOSPERM2 (FLO2) Confer Dull Grains with Low Amylose Content in Rice. Plant. Sci. 2015, 233, 44-52. [CrossRef]

43. Zhang, L.; Zhao, L.; Lin, L.; Zhao, L.; Liu, Q.; Wei, C. A Novel Mutation of OsPPDKB, Encoding Pyruvate Orthophosphate Dikinase, Affects Metabolism and Structure of Starch in the Rice Endosperm. Int. J. Mol. Sci. 2018, 19, 2268. [CrossRef] [PubMed]

Sample Availability: Samples of the starches are available from the authors.

(C) 2019 by the authors. Licensee MDPI, Basel, Switzerland. This article is an open access article distributed under the terms and conditions of the Creative Commons Attribution (CC BY) license (http://creativecommons.org/licenses/by/4.0/). 